autopsy was performed on each animal at the time of its death or when killed, and all were found to be free of tuberculous infection. Smears were made on slides from the spleens and right inguinal glands of all those pigs killed on the forty-first day, and examined microscopically for tubercle bacilli, and found to be negative in every instance, nor could tubercle bacilli be demonstrated in stained specimens of the centrifugalized washings before inoculation.

In the second experiment five articles of each sort were selected immediately after use and before washing of any kind, and swabbed with the 0.5 per cent. sodium bicarbonate solution as described above. These washings, which were very cloudy with particles of food, were immediately on arrival at the laboratory injected into eight guinea-pigs in the same manner as in the first experiment, the washings of each set of articles being divided between two pigs.

All these animals lived until the forty-first day, when they were killed and autopsied, and all, excepting the two inoculated with the washings from the milk glasses, were found to be tuberculous. It was an easy matter to demonstrate tubercle bacilli on slides made from the glands and spleens of these pigs, though no tubercle bacilli had been found in a casual search of smears made from the washings previous to inoculation.

The third experiment was a repetition of the first, but with the use of only six each of the utensils and twelve pigs, the washings from the different articles being distributed among three pigs. This test was made eight months later, when the dish-washing was being done by a new set of maids, but under the supervision of the same corps of nurses. The result, however, was the same.

It is fully realized that two experiments are not sufficient for definite conclusions, and that sterilization of eating utensils by heat or boiling, when possible, is not to be discouraged; at the same time when the dishwashing is thorough the danger of infection from this source does not seem to be very great.

My thanks are due to the Saranac Laboratory and the Reception Hospital for facilities placed at my disposal for the experiment, and to Dr. Baldwin, who suggested it.

121 Main Street.

\section{CONGENITAL INSUFFICIENCY OF THE PALATE *}

\section{G. W. STIMSON, A.B., M.D. ATLANTIC CITX, N. J.}

My attention was first directed to this condition by Makuen of Philadelphia, who exhibited a similar case before the College of Physicians and Surgeons about a year ago. As far as I have been able to discover, there has been no reference made to it in medical literature up to the present time.

Patient.-L. C., white, female, single, aged 18 , referred io me by Dr. Mary Townsend, of this city, consulted me Oet. 22, 1908. Practically her entire complaint was a marked defect, in speech, which she said had existed ever since she began to talk; it was that alone which prompted her to seek medical advice. There was slight nasal obstruction and some postnasal "dropping," but these were not troublesome enough to cause any complaint in themselves. There was no history of

\footnotetext{
* Read before the Atlantlc County Medical Society, Dec. 4, 1908.
}

diphtheria or other serious illness; in fact, with the exception of measles at the age of 14 , which clearly had had no effect on the condition under consideration, the patient had always enjoyed good health. The family history was negative.

Examination.-The palatal reflex was absent, and the free edge of the soft palate hung so far away from the posterior pharyngeal wall that a postnasal examination could be made with a large Iaryngeal mirror with perfect ease. Sensation was unimpaired. Occasionally small amounts of tea and coffee come through the nose, but apparently not often enough to be troublesome. The following points were further evidence of faulty development: The uvula was short and bifurcated. The palate, while not extremely high, was undoubtedly more highly arched than the average normal one. On palpation with the finger in the mouth, or on inspection while lifting the velum upward and backward with a blunt probe, it was seen that the posterior edge of the hard palate at the point of its attachment to the sott one, presented the shape of a long narrow letter $\mathrm{V}$ with the apex located well forward, and that the soft palate was abnormally short and not of sufficient length to reach the posterior pharyngeal wall. Incidentally, there was a hypertrophic rhinitis with a marked hypertrophy of the tips of the lower turbinals posteriorly, with mucoid degeneration of the mucous membrane covering them.

In normal yoice-production when any sounds except those of $m, n$, and $n g$ are spoken, the soft palate is made tense by the tensor palati muscle and lifted upward and backward by the levator palati until it comes tightly in contact with the posterior pharyngeal wall, thereby shutting off all communication with the nasal cavity and causing the oral cavity to be used as the chief resonatingchamber. But, when the sounds $m, n$ and $n g$ are produced, the soft palate hangs uncontracted, the communication with the nasal cavity remains intact and the latter plays the chief rôle as a resonating chamber.

In this case, so far as results are concerned, the palate is constantly in a state comparable to that of a normal individual during the production of these three sounds; that is, it is "insufficient" to shut out the nasal cavity as it should in the production of all other sounds, thereby allowing it to participate strongly as a resonating chamber not only in the production of the sounds of $\mathrm{m}$, $\mathrm{n}$ and $\mathrm{ng}$ as it should under normal conditions, and which, as would naturally be expected, she can say as plainly as anyone, but also in the production of all other sounds as well, a distinctly abnormal condition, thus imparting a strong nasal "twang" to her speech and causing her to talk through her nose.

In the development of the normal embryo, at about the fifth week the first visceral arch divides into an upper part, the maxillary arch, and a lower portion, the mandibular arch. The maxillary arches of the two sides unite at their anterior ends with the intervening nasofrontal process to form the upper boundary of the buccal cavity; the mandibulars to form the inferior boundary. In the fetus the palate process of the superior maxilla advances toward the median line until it meets and unites with its fellow of the opposite side. The horizontal plate of the palate bone develops similarly and very shortly after and thus is produced the hard palate. The two halves of the hard palate unite first in front, their union being completed about the twelfth week. Ordinarily it is stated that if union is incomplete the anomaly of cleft palate results.

In this case while union has apparently been practically complete and the patient has been fortunate enough to escape the greater evil of cleft palate, still development has not gone on to the perfection of completion (as is evidenced by the $\mathrm{V}$-shaped posterior edge and the high arch of the hard palate, the short soft pal- 
ate and the short and bifurcated uvula), but has been arrested in a stage which presents the above clinical picture, a condition to which Makuen very aptly gives the name of "congenital insufficiency of the palate."

100 st. Charles Place.

\section{IMPORTANCE OF URINE EXAMINATION IN SUSPECTED TYPHOID FEVER \\ CALVIN C. MARSHALL, M.D. PITTSBURG, PA.}

History.-I was first consulted in the case of Miss B., aged 19, November 29. She had headache, general malaise and loss of appetite, November 15, and these symptoms lasted for a week. At that time she was constipated and took two ounces of sodium and potassium tartrate. She then suffered from foul smelling diarrhea which persisted for three days and was followed by constipation. About November 22 the headache disappeared, though the general malaise remained, and that evening she had several chills, followed by a rise in temperature, for which she took three grains of quinin three times a day. Pain in the abdomen appeared November 26, and seemed to follow the transverse colon. It was of gradual onset and she applied a mustard plaster but without result.

Examination.-The patient was somewhat emaciated and very weak. The pupils were slightly dilated; the sclera was clear. The tongue had a heavy gray coating with a brown crusted center. The tip and edges were clear and red but not glazed. The breath was very foul. Temperature was $101 \mathrm{~F}$, pulse 88. Chest examination was negative.

Abdomen.-The abdomen was very muscular, flat, and some. what rigid. There was no gurgling. The patient complained of pain and tenderness in the region of the transverse colon; neither of these could be localized. Percussion and auscultation revealed nothing of value. The bowels had not moved for three days and the patient stated that the urine was darker than usual.

Diagnosis.-A fellow worker was in the hospital with typhoid and I made a provisional diagnosis of typhoid and told the patient to save a specimen of the next morning's urine. I examined the urine and found it loaded with bile. I again examined the abdomien and was able to palpate a distended gall bladder beneath the edge of an exceptionally large rectus muscle. There was localized pain and tenderness and I then mack? a diagnosis of cholangitis. Under appropriate treatment the condition cleared up in a week.

$\mathrm{Had}$ the urine not been examined in this case the patient might easily have been treated for typhoid, as there was no jaundice and the color of the stools was not noted. Had this been done, cholecystitis would probably have developed with its serious results for the patient. This case should emphasize the necessity for examination of the urine in all cases of typhoid or suspected typhoid.

439 Jucunda Street.

\section{Therapeutics}

\section{INDIVIDUAL TENDENCIES}

In this era of materialism the aversion to accepting anything as a fact, unless it can be occularly, aurally, chemically, microscopically or bacteriologically demonstrated, has relegated the "temperaments," diatheses, and even atavistic heritages, into the realm of oblivion. Sir Dyce Duckworth, in an address reported in the Lancet, March 7,1908 , calls the attention of the profession to the consideration of the factor of inheritance in the treatment of disease. For several years teachers of therapeutics have been endeavoring to teach the neces- sity of individualizing the patient, but even they have forgotten the necessity for also individualizing the family. Such individualization is not only of special advantage in the treatment of chronic conditions, but is also of advantage in the treatment of acute disease.

There is no more doubt that an individual inherits family weakness and family strength, or, if the phrase is preferred, family tendencies, than there is that he inherits the features and general physique of his parents and grandparents. While it may be going a step backward to speak of temperaments, we certainly should consider, as pointed out by Duckworth, the tendencies of the individual which may be enumerated as "arthritic," scrofulous or lymphatic, nervous, and bilious." These tendencies are often recognizable by the general appearance and physical findings but if not can almost always be developed by a careful investigation into the family history of the patient.

It should be the rule of the physician to inquire into the family history as carefully with every new patient as is required in an insurance examination. The discovery of instances of arteriosclerosis, cardiac disturbances, rheumatism, gout, or other joint disturbances in a family would show the arthritic tendency. The discovery of glandular disturbances, tuberculosis, pneumonias, adenoid and tonsillar disturbances, and anemias, in a family would show a tendency to "scrofulous" and lymphatic conditions which so frequently precede tuberculosis. While the word scrofulous has come into disuse and it may be a step backward to use it, Duckworth believes that it may be applied to the condition of childhood so long recognized under that name. With instances of neuroses and neurotic conditions, perhaps mental aberrations functional cardiac disturbances and nerve disturbances in the family, the patient will probably be neurotic and all symptoms should be considered accordingly. If the family shows a series of digestive troubles, liver, stomach, intestinal and kidney, the old term of "bilious temperament" or tendency may not be misapplied, if it is thoroughly understood what it signifies.

Duckworth well says "the physiologist has a dog or a guinea-pig, or some definite organ of an animal, but Jarely a man before him. The problems are not the same, and never can be. The personal factor, then, demands careful study from the physician, for men and women are not so many wooden ninepins turned in a lathe as some would have us believe. We have to reckon with seed and soil, not with seed only. Surely we have an exquisite analogy here in the sister vegetable kingdom which can not be ignored, viz.: the soil is as important to consider as the seed."

It is obviously certain that some families are predisposed to rheumatism and gout, for instance, and members of other families may be subjected to the same conditions and opportunities of infection (if. we believe that rheumatism is an infection) and still not be afflicted with these diseases. Thus it seems evident that there is a something present in one individual that will combat certain conditions and diseases which is not present in another individual who is really suffering from a predisposition to conditions of certain types. These predispositions or tendencies are perfectly evident in tuberculosis, the omnipresent tuherele bacilli heing constantly killed in certain individuals and readily living and thriving in others. Other persons with sickness, debilitv, orerwork and mental worry readily become nervous and mental' wrecks, while an individual 